\title{
Umbilical cord blood cells and brain stroke injury: bringing in fresh blood to address an old problem
}

\author{
Daniel A. Peterson
}

Department of Neuroscience, The Chicago Medical School at Rosalind Franklin University of Medicine and Science, North Chicago, Illinois, USA.

\begin{abstract}
Degeneration of brain tissue following stroke leads to functional impairment with limited brain self-repair. New evidence suggests that delivery of circulating $\mathrm{CD}^{+} 4^{+}$human umbilical cord blood cells can produce functional recovery in an animal stroke model with concurrent angiogenesis and neurogenesis leading to some restoration of cortical tissue (see the related article beginning on page 330). While some alternative interpretations of this data are offered herein, the study provides encouraging evidence of functional recovery from stroke in an animal model using stem cell therapy.
\end{abstract}

Focal brain ischemia results from blockage of the blood supply to the region supplied by the affected vessel with subsequent degeneration of that tissue. The severity of this degeneration is a function of the extent and location of the injury. There is little spontaneous repair of the injured region, and most post-stroke improvement appears to reflect the recruitment of intact circuitry to perform the function of the degenerated region. Because of the public health importance of developing an effective treatment leading to recovery from stroke, various animal models have been developed to simulate the mechanism of injury and to evaluate intervention strategies.

The observation that new neurons are generated in specific regions of the adult brain has raised the possibility that this process of neurogenesis may be harnessed to repopulate degenerated regions (1). Neurogenesis in the adult brain occurs in the hippocampal dentate gyrus and the forebrain subventricular zone (SVZ), it has been reported that new neurons can be generated either transiently or at a low rate in the cerebral cortex, substantia nigra, and hypothalamus (2-4), although the existence of cortical neurogenesis remains controversial (5). As a result, the adult brain can be divided into regions designated as neurogenic (dentate gyrus and forebrain SVZ) or nonneurogenic (the rest of the brain). However, recent evidence indicates that the forebrain SVZ of

Nonstandard abbreviations used: polysialylated neuronal cell adhesion molecule (PSA-NCAM); subventricular zone (SVZ).

Conflict of interest: The author engages in private consultation activity through NeuroRenew Inc.

Citation for this article: J. Clin. Invest. 114:312-314 (2004). doi:10.1172/JCI200422540. humans does not give rise to new neurons, suggesting that in the human brain, only the dentate gyrus may support substantial amounts of neurogenesis (6). Such restriction in the human brain may have implications for the therapeutic use of neural stem cells. Nevertheless, in animal models, neural stem cells can be extracted from various brain regions and expanded in vitro (7). Recent studies suggest that cells expressing the chondroitin sulphate proteoglycan NG2, thought to serve as a marker for neural progenitors committed to an oligodendrocyte lineage, may exhibit multipotentiality in vitro $(8,9)$. As NG2-positive cells are widespread throughout the mature brain, they may represent a local reserve of stem cells that may be recruited for repair of focal injury.

Within neurogenic regions, the rate of neurogenesis appears to be modulated by local environmental changes (10). Neurogenesis can also be promoted by the delivery to neurogenic regions of various growth factors, including brain-derived neurotrophic factor, FGF-2, IGF, and erythropoietin (11-13). Following experimental injury, there is an upregulation of neurogenesis, and delivery of growth factors in injury models can augment this neurogenic response as well as contribute to neuroprotection, which suggests that this may be a suitable strategy to pursue for development of therapeutic intervention (14, 15). Specific enhancement of neurogenesis has been reported as a result of ischemic injury with migration of neuroblasts to the site of degeneration, although only a small fraction of these new neurons survive (reviewed in ref. 16).

It is not yet understood what environmental differences between neurogenic and nonneurogenic brain regions support the gener- ation of new neurons, but this process may be regulated by the availability of factors to enhance neurogenesis, the presence of factors that suppress neurogenesis, or a combination of the two. The situation is made more complex by the progression of cells from proliferation through lineage commitment and functional differentiation, all of which are sequential components in the process of neurogenesis. Furthermore, cell progression through each of these components of neurogenesis is likely to be regulated by different factors at each step. Thus, the development of successful therapeutic strategies to use neural stem cells for brain repair will require that appropriate regulatory factors be presented in the proper spatial and temporal sequence. This is a daunting prospect, and progress will come as individual pieces of this puzzle are assembled.

\section{Cord blood cells promote stroke recovery}

The report by Taguchi et al. in the current issue of the JCI (17) offers one piece of this puzzle by showing that intravenous delivery of the $\mathrm{CD} 34^{+}$subpopulation of human umbilical cord blood cells can enhance angiogenesis, neurogenesis, and morphological and functional recovery in an animal model of stroke (Figure 1). Behavioral recovery from stroke and injury following delivery of human umbilical cord blood cells has been observed, but in the absence of significant numbers of grafted cells entering the brain, it was not previously clear how this effect was mediated (18-20). Here Taguchi et al. show that the peripheral cells may themselves show limited incorporation but serve to stimulate angiogenesis and perhaps lift suppression of neurogenesis (possibly by the same mechanism of repressor inhibition that leads to angiogenesis). Following induction of a middle cerebral artery occlusion model of stroke, mice receiving $\mathrm{CD} 34^{+}$cord blood cells within 48 hours of the insult demonstrated significant improvement on relevant behavioral tests and a modest, but significant, increase in cortical thickness. Following substantial angiogenesis in regions surrounding the inju- 


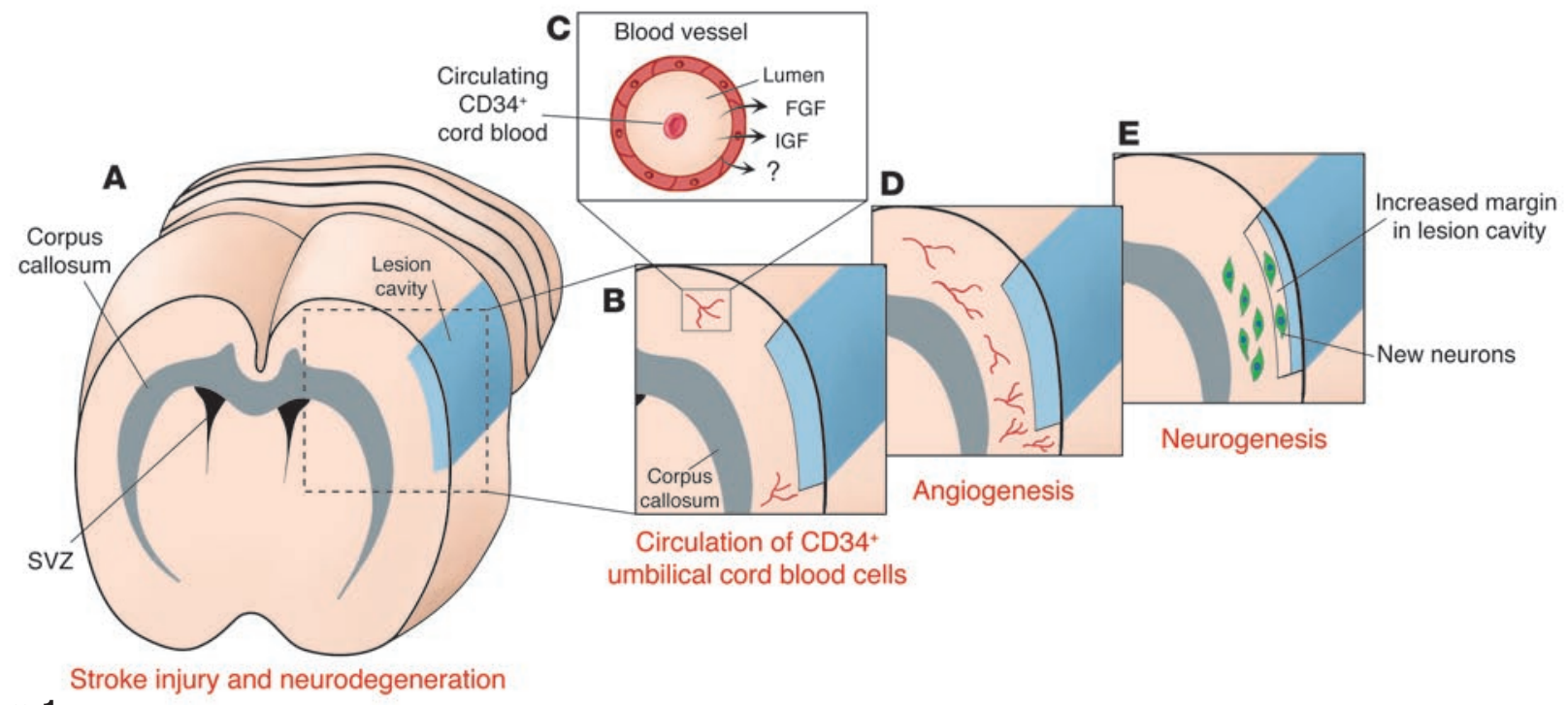

Figure 1

Morphological response of stroke-injured brain to delivery of umbilical cord blood cells. (A) Occlusive stroke results in the degeneration of brain tissue supplied by the occluded vessel, which produces a lesion cavity. The lesion cavity compromises the cerebral cortex and lies some distance from a neurogenic region, the SVZ. The major fiber tracts forming the corpus callosum lie between the lesion cavity and the SVZ, a putative source of newly generated neurons observed in response to stem cell therapy. (B) Delivery of umbilical cord blood cells enriched with the CD34+ cell fraction to the tail vein results in circulation of these primitive stem cells throughout the body's vascular system. (C) The circulating CD34+ cord blood cells in vessels adjacent to regions of neurodegeneration provide a possible supply of a number of cytokines and growth factors, including FGF-2 and IGF, that are proposed to cross into brain parenchyma directly or to stimulate local environmental production of growth factors. (D) Factors released by the circulating CD $34^{+}$cells also serve to recruit angioblasts, leading to angiogenesis. (E) Following CD $34^{+}$cord blood cell delivery, new cells are born and express neuronal lineage markers in a process called neurogenesis. In addition to being present in tissue remaining after the lesion, these new neurons contribute to restored tissue in the margin of the lesion cavity, which results in a small lesion cavity. It remains to be determined whether the newly generated neurons migrated from the neurogenic SVZ or were recruited from quiescent neural progenitor or stem cells residing in the parenchyma closer to the lesion cavity. Figure adapted with permission from Current Opinion in Neurobiology (16).

ry, animals that received the cord blood cells showed a number of migrating neuroblasts in the margin of the cortical injury, primarily on the aspect closest to the forebrain SVZ. Over time, the cortex at the site of injury increased in thickness relative to measurements made shortly after degeneration had occurred. Unfortunately, systematic sampling with stereological measures was not employed; thus the extent of morphological response is unclear as only a few samples were measured for linear changes in cortical thickness and density of cells. The authors concluded that the $\mathrm{CD}_{3} 4^{+}$cells in the cord blood stimulated angiogenesis with specific neovascularization around the cortical degeneration. In turn, neurogenesis was stimulated by these events with subsequent migration of neuroblasts into the newly restored cortex where these cells matured and were responsible for functional recovery.

\section{Stem cell therapy with cord blood: how might it work?}

These are exciting findings with direct therapeutic possibilities, but there are alternative interpretations for some aspects of the study by Taguchi et al. (17). The authors attribute the behavioral recovery of the mice to the functioning of newly generated neurons. However, the improvement in cortical thickness was quite modest. By 90 days, animals receiving $\mathrm{CD}_{3} 4^{+}$cells had a $25 \%$ thicker cortex than non-CD $34^{+}$-treated animals or had $15 \%$ less loss of cortical thickness, depending on the context in which the results are viewed. It is difficult to see how such a limited morphological increase could account for functional restoration, particularly in the absence of data revealing the morphological and functional connectivity of new cells. It is also possible that the neovascularization the authors observed may have benefited uncompromised tissue, leading to enhanced behavioral performance. Further studies, in which neurogenesis is specifically blocked, will be needed to provide insight into the role of neurogenesis and to validate the authors' conclusion that behavioral improvement was due to functional neurogenesis.

Taguchi et al. (17) also assume that the new neurons have migrated from the forebrain SVZ because of their expression of the migratory markers polysialylated neuronal cell adhesion molecule (PSA-NCAM) and doublecortin. While these are both mark- ers of migrating neuroblasts, their expression does not provide information about the extent of their migration. For example, expression of PSA-NCAM and doublecortin are also found in the new neurons in the dentate gyrus where the total migratory movement is only a few cell diameters. These cells may be migrating, but they need not have come all the way from the forebrain SVZ. It may be that these cells were recruited from local progenitor cells, such as the NG2positive cell population described earlier. For example, in the immediate post-stroke interval, substantial cell proliferation was observed near the site of injury and $25 \%$ of these cells were identified as adopting an endothelial cell fate. However, the ultimate identity of the remaining BrdU-positive cells was not pursued, and it is possible that these may have eventually expressed neuronal lineage markers in response to signals associated with CD34+ cells and neovascularization.

It would be interesting to know what effect the stimulation provided by circulating $\mathrm{CD}_{34}{ }^{+}$umbilical cord blood cells would have on neurogenesis in the forebrain SVZ and dentate gyrus regions of naive and stroke-lesioned animals. Such information 
would be useful for discriminating between the influence of CD34 $4^{+}$cells and that of neovascularization on the overall neurogenic capacity of the dentate gyrus and forebrain SVZ. It may also provide insight into the possibility of recruiting neural stem cells from these regions for brain repair. This information would be particularly relevant in regard to the experimental group that also received erythropoietin, as this treatment has been shown to enhance both angiogenesis and neurogenesis (13). The current study suggests that one mechanism accounting for the functional recovery resulting from cord blood cell delivery works through the enhancement of angiogenesis around the site of degeneration. This finding provides a note of optimism for developing therapeutic strategies for stroke. Taguchi et al. provide an important piece of the puzzle, but there remains much to be determined about the mechanisms involved and the specific role of neurogenesis in brain recovery from stroke before a comprehensive picture will emerge on how to treat this old problem (17).

\section{Acknowledgments}

The author's research is supported in part by NIH grants AG20047 and AG22555.

Address correspondence to: Daniel A. Peterson, Department of Neuroscience,
The Chicago Medical School at Rosalind Franklin University of Medicine and Science, 3333 Green Bay Road, North Chicago, Illinois 60064, USA. Phone: (847) 578-3411; Fax: (847) 578-8545; E-mail: daniel.peterson@rosalindfranklin.edu.

1. Hallbergson, A.F., Gnatenco, C., and Peterson, D.A. 2003. Neurogenesis and brain injury: managing a renewable resource for repair. J. Clin. Invest. 112:1128-1133. doi:10.1172/JCI200320098.

2. Gould, E., Vail, N., Wagers, M., and Gross, C.G. 2001. Adult-generated hippocampal and neocortical neurons in macaques have a transient existence. Proc. Natl. Acad. Sci. U. S. A. 98:10910-10917.

3. Zhao, M., et al. 2003. Evidence for neurogenesis in the adult mammalian substantia nigra. Proc. Natl. Acad. Sci. U. S. A. 100:7925-7930.

4. Markakis, E.A., Palmer, T.D., Randolph-Moore, L., Rakic, P., and Gage, F.H. 2004. Novel neuronal phenotypes from neural progenitor cells. J. Neurosci. 24:2886-2897.

5. Kornack, D.R., and Rakic, P. 2001. Cell proliferation without neurogenesis in adult primate neocortex. Science. 294:2127-2130.

6. Sanai, N., et al. 2004. Unique astrocyte ribbon in adult human brain contains neural stem cells but lacks chain migration. Nature. 427:740-744.

7. Palmer, T.D., Markakis, E.A., Willhoite, A.R., Safar, F., and Gage, F.H. 1999. Fibroblast growth factor-2 activates a latent neurogenic program in neural stem cells from diverse regions of the adult CNS. J. Neurosci. 19:8487-8497.

8. Nunes, M.C., et al. 2003. Identification and isolation of multipotential neural progenitor cells from the subcortical white matter of the adult human brain. Nat. Med. 9:439-447.

9. Belachew, S., et al. 2003. Postnatal NG2 proteoglycan-expressing progenitor cells are intrinsically multipotent and generate functional neurons. J. Cell Biol. 161:169-186.

10. Peterson, D.A. 2002. Stem cells in brain plasticity and repair. Curr. Opin. Pharmacol. 2:34-42.

11. Pencea, V., Bingaman, K.D., Wiegand, S.J., and Luskin, M.B. 2001. Infusion of brain-derived neurotrophic factor into the lateral ventricle of the adult rat leads to new neurons in the parenchyma of the striatum, septum, thalamus, and hypothalamus. J. Neurosci. 21:6706-6717.

12. Yoshimura, S., et al. 2001. FGF-2 regulation of neurogenesis in adult hippocampus after brain injury. Proc. Natl. Acad. Sci. U. S. A. 98:5874-5879.

13. Shingo, T., Sorokan, S.T., Shimazaki, T., and Weiss, S. 2001. Erythropoietin regulates the in vitro and in vivo production of neuronal progenitors by mammalian forebrain neural stem cells. J. Neurosci. 21:9733-9743.

14. Yoshimura, S., et al. 2003. FGF-2 regulates neurogenesis and degeneration in the dentate gyrus after traumatic brain injury in mice. J. Clin. Invest. 112:1202-1210. doi:10.1172/JCI200316618.

15. Nakatomi, H., et al. 2002. Regeneration of hippocampal pyramidal neurons after ischemic brain injury by recruitment of endogenous neural progenitors. Cell. 110:429-441.

16. Kokaia, Z. and Lindvall, O. 2003. Neurogenesis after ischaemic brain insults. Curr. Opin. Neurobiol. 13:127-132.

17. Taguchi, A., et al. 2004. Administration of CD34+ cells after stroke enhances neurogenesis via angiogenesis in a mouse model. J. Clin. Invest. 114:330-338. doi:10.1172/JCI200420622.

18. Chen, J., et al. 2001. Intravenous administration of human umbilical cord blood reduces behavioral deficits after stroke in rats. Stroke. 32:2682-2688.

19. Willing, A.E., et al. 2003. Intravenous versus intrastriatal cord blood administration in a rodent model of stroke. J. Neurosci. Res. 73:296-307.

20. Saporta, S., et al. 2003. Human umbilical cord blood stem cells infusion in spinal cord injury: engraftment and beneficial influence on behavior. J. Hematother. Stem Cell Res. 12:271-278.

\title{
Unlocking the secrets of the pancreatic $\beta$ cell: man and mouse provide the key
}

\author{
Andrew T. Hattersley \\ Diabetes and Vascular Medicine, Institute of Biomedical and Clinical Science, Peninsula Medical School, Exeter, United Kingdom.
}

\begin{abstract}
Failure of the pancreas to secrete sufficient insulin results in type 2 diabetes, but the pathogenesis of pancreatic $\beta$ cell dysfunction is still poorly understood. New insights into $\beta$ cell failure come from defining the genes involved in rare genetic subtypes of diabetes and creating appropriate animal models. A new mouse model of transient neonatal diabetes mellitus emphasizes that both the number of $\beta$ cells and their function are critical for insulin secretion and may be regulated by imprinted genes (see the related article beginning on page 339).
\end{abstract}

Nonstandard abbreviations used: hydatidiform mole-associated and -imprinted transcript (HYMAI); P1-derived artificial chromosome (PAC); transient neonatal diabetes mellitus (TNDM); type 2 diabetes (T2D); $\underline{Z}$ finger protein that regulates apoptosis and cell cycle arrest $(Z A C)$.

Conflict of interest: The author has declared that no conflict of interest exists.

Citation for this article: J. Clin. Invest. 114:314-316 (2004). doi:10.1172/JCI200422506.
The regulated secretion of insulin by the pancreatic $\beta$ cell maintains blood sugar concentrations within a narrow physiological range. In over 150 million people worldwide, however, pancreatic $\beta$ cells fail to secrete adequate insulin, usually in the presence of increased insulin resistance, which results in type 2 diabetes (T2D). Understanding the pathways that result in $\beta$ cell dysfunction at a physiological and molecular level is critical for improved understanding and treatment of T2D.

\section{Learning from rare genetic subtypes of diabetes}

How can we study the pancreatic $\beta$ cell in humans when these cells are not readily accessible? Accidents of nature in which a single gene defect results in severe $\beta$ cell dysfunction, causing diabetes, offer the chance of gaining new insights into this disease if the responsible gene can be defined. The best example of such research has been the use of positional cloning to demonstrate that heterozygous mutations of the genes encoding the hepatic transcription factors 